\title{
Prognostic significance and tumor-immune infiltration of mTOR in clear cell renal cell carcinoma
}

\author{
Na Li ${ }^{1}$, Jie Chen ${ }^{1}$, Qiang Liu ${ }^{2}$, Hongyi Qu ${ }^{3}$, Xiaoqing Yang ${ }^{4}$, Peng Gao ${ }^{2}$, Yao Wang ${ }^{2}$, Huayu Gao $^{3}$, Hong Wang \\ Corresp., 1 , Zuohui Zhao ${ }^{\text {Corresp. } 3}$ \\ ${ }^{1}$ Department of Urology, The First Affiliated Hospital of Shandong First Medical University \& Shandong Provincial Qianfoshan Hospital, Jinan, Shandong, \\ China \\ 2 Department of Cardiology, The First Affiliated Hospital of Shandong First Medical University \& Shandong Provincial Qianfoshan Hospital, Jinan, Shandong, \\ China \\ 3 Department of Pediatric Surgery, The First Affiliated Hospital of Shandong First Medical University \& Shandong Provincial Qianfoshan Hospital, Jinan, \\ Shandong, China \\ 4 Department of Pathology, The First Affiliated Hospital of Shandong First Medical University \& Shandong Provincial Qianfoshan Hospital, Jinan, Shandong, \\ China
}

Corresponding Authors: Hong Wang, Zuohui Zhao

Email address: wanghong@sdhospital.com.cn, zhaozuohui@126.com

Mammalian target of rapamycin (mTOR), a serine/threonine kinase involved in cell proliferation, survival, metabolism and immunity, was reportedly activated in various cancers. However, the clinical role of mTOR in renal cell carcinoma (RCC) is controversial. Here we detected the expression and prognosis of total MTOR and phosphorylated mTOR ( $p$-mTOR) in clear cell RCC (ccRCC) patients, and explored the interactions between mTOR and immune infiltrates in ccRCC. The protein level of mTOR and $p$-mTORwas determinedby western blotting (WB), and their expression was evaluated in $145 \mathrm{ccRCC}$ and 13 non-tumor specimens by immunohistochemistry (IHC). The relationship to immune infiltration of mTOR was further investigated using TIMER and TISIDB databases, respectively. WB demonstrated the ratio of $p-m T O R$ to $m$ TOR was higher in $c c R C C$ than adjacent specimens $(n=3)$, and IHC analysis elucidated that $p$-mTOR expression was positively correlated with tumor size, stage and metastasis status, and reversely correlated with cancer-specific survival (CSS). In univariate analysis, high grade, large tumor, advanced stage, metastasis, and high p-mTOR expression were recognized as prognostic factors of poorer CSS, and multivariate survival analysis elucidated that tumor stage, $p$-mTOR and metastasis were of prognostic value for CSS in CCRCC patients. Further TIMER and TISIDB analyses uncovered that mTOR gene expression was significantly associated with numerous immune cells and immunoinhibitors in patients with ccRCC. Collectively, these findings revealed p-mTOR was identified as an independent predictor of poor survival, and mTOR was associated with tumor immune infiltrates in ccRCC patients, which validated mTOR could be implicated in the initiation and progression of $c \mathrm{RCC}$. 


\section{Prognostic significance and tumor-immune infiltration of}

\section{2 mTOR in clear cell renal cell carcinoma}

3

$4 \mathrm{Na} \mathrm{Li}^{1}$, Jie Chen ${ }^{1}$, Qiang Liu ${ }^{2}$, Hongyi Qu ${ }^{3}$, Xiaoqing Yang ${ }^{4}$, Peng Gao ${ }^{2}$, Yao Wang ${ }^{2}$, Huayu

5 Gao $^{3}$, Hong Wang ${ }^{1 *}$, Zuohui Zhao ${ }^{3 *}$

6

$7{ }^{1}$ Department of Urology, The First Affiliated Hospital of Shandong First Medical University \&

8 Shandong Provincial Qianfoshan Hospital, Jinan, Shandong, China

$9{ }^{2}$ Department of Cardiology, The First Affiliated Hospital of Shandong First Medical University

10 \& Shandong Provincial Qianfoshan Hospital, Jinan, Shandong, China

$11{ }^{3}$ Department of Pediatric Surgery, The First Affiliated Hospital of Shandong First Medical

12 University \& Shandong Provincial Qianfoshan Hospital, Jinan, Shandong, China

$13{ }^{4}$ Department of Pathology, The First Affiliated Hospital of Shandong First Medical University \&

14 Shandong Provincial Qianfoshan Hospital, Jinan, Shandong, China

16 Corresponding Author:

17 Hong Wang, Email address: wanghong@sdhospital.com.cn;

18 Zuohui Zhao, Email address: zhaozuohui@126.com. 


\section{Abstract}

21 Mammalian target of rapamycin (mTOR), a serine/threonine kinase involved in cell

22 proliferation, survival, metabolism and immunity, was reportedly activated in various cancers.

23 However, the clinical role of mTOR in renal cell carcinoma (RCC) is controversial. Here we

24 detected the expression and prognosis of total mTOR and phosphorylated mTOR (p-mTOR) in

25 clear cell RCC (ccRCC) patients, and explored the interactions between mTOR and immune

26 infiltrates in ccRCC. The protein level of mTOR and p-mTOR was determined by western

27 blotting (WB), and their expression was evaluated in $145 \mathrm{ccRCC}$ and 13 non-tumor specimens

28 by immunohistochemistry (IHC). The relationship to immune infiltration of mTOR was further

29 investigated using TIMER and TISIDB databases, respectively. WB demonstrated the ratio of p-

30 mTOR to mTOR was higher in ccRCC than adjacent specimens $(n=3)$, and IHC analysis

31 elucidated that p-mTOR expression was positively correlated with tumor size, stage and

32 metastasis status, and reversely correlated with cancer-specific survival (CSS). In univariate

33 analysis, high grade, large tumor, advanced stage, metastasis, and high p-mTOR expression were

34 recognized as prognostic factors of poorer CSS, and multivariate survival analysis elucidated that

35 tumor stage, p-mTOR and metastasis were of prognostic value for CSS in ccRCC patients.

36 Further TIMER and TISIDB analyses uncovered that mTOR gene expression was significantly

37 associated with numerous immune cells and immunoinhibitors in patients with ccRCC.

38 Collectively, these findings revealed p-mTOR was identified as an independent predictor of poor

39 survival, and mTOR was associated with tumor immune infiltrates in ccRCC patients, which

40 validated mTOR could be implicated in the initiation and progression of ccRCC. 


\section{Introduction}

43 Cancer has become the first or second leading cause of death and one major burden worldwide

44 nowadays (Siegel et al. 2021; Sung et al. 2021). Kidney cancer is a common urological

45 neoplasm, with estimated 431,288 newly diagnosed cases and 179,368 cancer-related deaths

46 worldwide in 2020 (Sung et al. 2021). Renal cell carcinoma (RCC), the most common

47 malignancy within the kidney, comprises a heterogeneous group of tumors including clear cell

48 (70 80\%), papillary (10 15\%), chromophobe (3 5\%), and other less common subtypes (Rini et

49 al. 2009). Radical nephrectomy is the gold standard for localized RCC, while systemic therapy is

50 the main course of treatment for patients with advanced RCC, especially metastatic RCC

51 (mRCC) (Posadas et al. 2017). As RCC is resistant to traditional chemotherapy and radiotherapy,

52 medical therapy for $\mathrm{mRCC}$ has transitioned from a nonspecific immune approach (cytokine

53 therapy) to novel molecular targeted therapy, including tyrosine kinase inhibitors (TKIs),

54 mammalian target of rapamycin (mTOR) inhibitors, and immune checkpoint inhibitors (Barata \&

55 Rini 2017). Multiple TKIs, especially Sunitinib, are widely used pharmacologic agents for

56 mRCC patients (Posadas et al. 2017). Two mTOR inhibitors, Temsirolimus as a first-line therapy

57 and Everolimus as a second-line therapy, have been approved for clinical use in mRCC patients.

58 Immune checkpoint inhibitors such as programmed cell death protein 1 (PD-1) inhibitor,

59 Nivolumab and programmed death-ligand 1(PD-L1) inhibitor Avelumab, directly reverse the

60 adaptive tumor cell deploy to avoid host immunity, thus attenuate immune response and

61 postpone cancer progression (Makhov et al. 2018; Posadas et al. 2017). However, molecular

62 targeted therapy for RCC remains limited due to varied response rate and apparent adverse

63 effects, such as fatigue, diarrhoea and hyperglycaemia (Posadas et al. 2017). Of particular note,

64 the mechanisms underlying the different responsiveness and side-effect might be complicated.

65 Combined therapy with multiple molecular-targeted drugs has attracted much attention (Posadas

66 et al. 2017). Thus, there is an urgent requirement to further investigate the molecular

67 mechanisms that drive RCC initiation and progression, which would facilitate to realize

68 individual or personalized therapy for this heterogeneous tumor. 
69 mTOR, a highly conserved serine/threonine kinase, is a core component of phosphatidylinositol 703 kinase (PI3K)/AKT/mTOR signaling pathway. mTOR encompasses two functionally distinct 71 protein complexes: mTOR complex 1 (mTORC1) and mTORC2. mTORC1 contains rapamycin72 sensitive RAPTOR (regulatory associated protein of mTOR), while mTORC2 contains 73 rapamycin-insensitive companion of mTOR (RICTOR). mTOR regulates diverse biological 74 processes, including cell proliferation, survival, metabolism, and immunity under physiological 75 and pathological conditions (Posadas et al. 2017; Saxton \& Sabatini 2017). mTOR is generally 76 activated (characterized by phosphorylation at Ser2,448) to catalyze the phosphorylation of its 77 downstream targets and modulate their activity, such as ribosomal S6 protein kinase 1 (S6K1), 78 eukaryotic translation initiation factor $4 \mathrm{E}$ binding protein $1(4 \mathrm{E}-\mathrm{BP} 1)$, and protein kinase $\mathrm{C}$ 79 (PKC), thereby regulating protein synthesis, cell growth, and metabolism (Posadas et al. 2017). 80 Recent researches also focus on its role in cancer. mTOR was reported to be aberrantly activated 81 during tumorigenesis, and it was found to have played a crucial role in the initiation and 82 progression of various malignant tumors, including breast, prostate, lung, liver, and kidney 83 cancers (Posadas et al. 2017). Due to its characterized activity, mTOR has been recognized as a 84 target for cancer therapy.

85 The advent of high throughput technologies and massive public databases, such as The Cancer 86 Proteome Atlas (TCPA), The Cancer Genome Atlas (TCGA), and some online cancer genome 87 tools, such as Tumor Immune Estimation Resource (TIMER), Tumor-Immune System 88 Interactions and DrugBank (TISIDB), have facilitated to elaborate the complex molecular 89 mechanism in cancer (Li et al. 2013; Li et al. 2017; Ru et al. 2019). Previous studies 90 demonstrated that phosphorylated mTOR (p-mTOR) was increased in RCC, and enhanced p91 mTOR was associated with impaired overall survival (OS), which indicated that the $92 \mathrm{PI} 3 \mathrm{~K} / \mathrm{AKT} / \mathrm{mTOR}$ signaling pathway could promote the initiation and progression of RCC 93 (Darwish et al. 2013; Kruck et al. 2010; Liontos et al. 2017; Rausch et al. 2019). Recent large94 scale proteogenomic analyses found that mTOR and/or p-mTOR expression levels were 95 significantly correlated with improved outcomes in RCC (Fan et al. 2020; Zhang et al. 2017), 
96

97

98

99

100

101

102

103

104

105

106

107

108

109

110

111

112

113

114

115

116

117

118

119

120

121

which was contradictory to the most widely accepted research and data. Collectively, the above studies failed to reach a consistent conclusion, and the prognostic role of $\mathrm{mTOR}$ in RCC remains controversial. In the current study, we detected total mTOR and p-mTOR expression in 3 pairs clear cell RCC (ccRCC) and their corresponding non-tumor kidney specimens using western blotting (WB), examined mTOR (including p-mTOR) expression in 145 ccRCC and 13 adjacent nonneoplastic kidney specimens using immunohistochemistry (IHC) with tissue microarray (TMA), explored the relationship between mTOR (including p-mTOR) expression and the clinicopathological parameters (including patients' age and tumor size), and elucidated the complex tumor-immune interactions in ccRCC using TIMER and TISIDB databases, which tried to investigate the clinical role of mTOR and p-mTOR in ccRCC patients.

\section{Materials \& Methods}

Patients. The research was approved by the ethical committees of The First Affiliated Hospital of Shandong First Medical University (2017-S007), and all the participants signed the informed consents. Totally 148 ccRCC patients with resectable tumors between March 2010 and January 2015 were enrolled (Yuan et al. 2020). All the ccRCC samples were primary lesions, and they were verified using hematoxylin and eosin (HE) staining by two pathologists (XQ Yang and Y

Wang) after surgery. The cohort \#1 was used to compare mTOR and p-mTOR expression by WB analysis, which consisted of 3 cases of ccRCC and their corresponding normal kidney specimens ( 1 female and 2 males, age from 55 to 65, International Society of Urological Pathology (ISUP) grading with $1 \mathrm{G} 1+1 \mathrm{G} 2+1 \mathrm{G} 3$, American Joint Committee on Cancer (AJCC) staging with 1 TI +2 TII]. The cohort \#2 was used to evaluate the prognostic value of mTOR and p-mTOR by IHC assay. TMA from 145 ccRCC and 13 non-tumor specimens was created as described previously (Yuan et al. 2020). The patient characteristics (such as tumor grade, stage, patients' sex, and age) and follow-up period were recorded (Table 1). The median period of follow-up was 47.2 months, ranging from 20 to 76 months. Among the HE-stained slides, 10 archived paraffinembedded tissue sections (including 4 females and 6 males, age from 45 to 68, ISUP grading 
122 with $2 \mathrm{G} 1+5 \mathrm{G} 2+3 \mathrm{G} 3$, AJCC staging with $3 \mathrm{TI}+6 \mathrm{TII}+1 \mathrm{TIII})$ were randomly selected and 123 used to examine the immune cells.

124 WB analysis. Three pairs ccRCC and adjacent tissues were chosen for WB analysis (Yuan et al. 125 2020). Membranes were incubated with the desired primary antibodies: mTOR (1:2,000, rabbit, 126 ab32028; Abcam, Cambridge, MA), p-mTOR (Ser2448, 1:2,000, rabbit, ab109268; Abcam, 127 Cambridge, MA), anti-Actin (1:1,000, mouse, BM0627; BOSTER, Beijing, China), the 128 corresponding secondary antibodies (SA00001-2 \& SA00001-1; Proteintech, Wuhan, China) 129 were diluted to 1:5,000, and enhanced chemiluminescence (Amersham Imager 600, 130 Marlborough, MA) was used for immunodetection as previously described (Yuan et al. 2020). 131 IHC analysis. TMA was constructed from $145 \mathrm{ccRCC}$ and 13 adjacent kidney specimens, which 132 was used for IHC analysis as previously described (Zhao et al. 2016). The slides were stained 133 with the described primary antibodies: mTOR (1:100) and p-mTOR (1:100), and 134 diaminobenzidine (ZLI-9017; Zhongshan, Beijing, China) was used for visualization. Human 135 placenta served as negative controls (Rausch et al. 2019).

136 The staining was analyzed by two independent reviewers who were unaware of the disease 137 outcome. The expression level of mTOR and p-mTOR was evaluated and scored according to 138 their staining intensity (0 3) and frequency (0 4) as previously described (Yuan et al. 2020). 139 Accordingly, they were categorized into 2 groups: high group (staining scores $\geq 3$ for $\mathrm{p}$-mTOR, $140 \geq 2$ for mTOR) and low group (scores $<3$ for $\mathrm{p}-\mathrm{mTOR},<2$ for $\mathrm{mTOR}$ ).

141 TIMER database analysis. TIMER database (https://cistrome.shinyapps.io/timer) was used 142 to explore the association between mTOR gene expression and immune cell infiltrates of ccRCC, 143 which estimated the abundance of tumor-infiltrating immune cells (TIICs) from TCGA, as 144 previously described (Li et al. 2017). In brief, Gene module was chosen to analyze the complex 145 tumor-immune interactions between mTOR and six TIIC subsets, i.e., B cells, CD4 +T cells, $146 \mathrm{CD} 8+\mathrm{T}$ cells, macrophages, neutrophils, and dendritic cells, in the ccRCC cohort ( $\mathrm{n}=533)$.

147 purity adjusted was selected, and the scatter plots of Spearman's correlations between them were 148 displayed. 
149 TISIDB database analysis. TISIDB database (http://cis.hku.hk/TISIDB), which integrated 150 multiple public databases including TCGA, was also used to reveal the immune infiltration of 151 mTOR in cancer, as previously described (Ru et al. 2019). In this study, Lymphocyte and 152 Immunomodulator modules were selected to investigated the correlations between mTOR gene 153 expression and abundance of tumor-infiltrating lymphocytes (TILs) \& immunoinhibitors in $\operatorname{ccRCC}(\mathrm{n}=534)$, then the heatmaps and scatter plots between mTOR and 28 TILs \& 24 immunoinhibitors from Charoentong's study, were displayed subsequently.

Statistical analysis. All analysis was carried out using SPSS 21.0 software (SPSS Inc., Chicago, IL, USA). For WB analysis, the differential expression of mTOR and p-mTOR was compared by means of Student's t-test. Correlations between mTOR (including p-mTOR) expression and clinical parameters were calculated using Pearson Chi-square test. The survival curves for cancer-specific survival (CSS) were calculated using Kaplan-Meier method, and prognostic factors were analyzed using Cox proportional hazard regression model. For TIMER and TISIDB analyses, Spearman's correlations analysis was used to infer the association between mTOR and tumor immune infiltrates. $P<0.05$ was considered statistically significant.

\section{Results}

mTOR and p-m TOR expression in ccRCC. First, we compared the protein expression of $\mathrm{mTOR}$ and p-mTOR between 3 cases of ccRCC and their adjacent non-tumor specimens. WB demonstrated that the expression level of mTOR showed a decreasing trend in ccRCC compared with adjacent specimens ( $P=0.077$, Fig. 1$)$, which was consistent with its mRNA expression (Fan et al. 2020). While p-mTOR expression was stable in both malignant and adjacent nontumor tissues $(P=0.894)$. When normalized to mTOR expression, we found the ratio of $\mathrm{p}$-mTOR to mTOR elevated 4.259 folds in cancerous than adjacent kidney specimens $(P=0.032)$, which validated previous report (Kruck et al. 2010).

\section{The relationship between $m$ TOR (including p-mTOR) expression and clinicopathological}

parameters of $\boldsymbol{c} \boldsymbol{C R} \boldsymbol{C} \boldsymbol{C}$. Then we analyzed mTOR (including p-mTOR) expression and its association with patient characteristics (Table 1). IHC analysis on TMA showed that mTOR positive signal was distributed in majority of the adjacent non-tumor kidney tubule epithelial cells and sparse malignant cells, and p-mTOR positive signal was aggregately scattered in nonneoplastic kidney tubule epithelial cells and cancer cells (Fig. 2). Relative weak cytoplasm 
179 staining for mTOR and strong staining for $\mathrm{p}$-mTOR expression were seen in the malignant cells

180 of kidney. To be specific, stronger positive staining with p-mTOR was examined in 73 (50.34\%) 181 cases and weaker staining was examined in 72 (49.66\%) cases of ccRCC tissues, respectively. p182 mTOR expression was higher in large tumors $(\geq 7.0 \mathrm{~cm})$ than small ones $(<7.0 \mathrm{~cm})$, the 183 difference was statistically significant $(P=0.001)$. Moreover, enhanced p-mTOR expression $(\geq 3$ 184 scores) was positively correlated with advanced stage (stage III-IV, $P<0.001$ ) and metastatic 185 status (M1, $P=0.003)$, and it was reversely correlated with CSS rate $(P<0.001)$. The 186 relationship between p-mTOR expression and patients' sex, age or tumor grade was not 187 significant $(P=0.056, P=0.244, P=0.397$, respectively). As for mTOR, its enhanced 188 expression ( $\geq 2$ scores) was not significantly correlated with any clinicopathological parameters such as tumor grade, stage, size, metastasis, survival rate, patients' sex or age $(P>0.05)$, which 190 was also observed in previous study (Rausch et al. 2019). To sum up, the above data elucidated that $\mathrm{p}$-mTOR expression was positively correlated with tumor size, pathological stage and metastasis status, and reversely correlated with CSS, which indicated that p-mTOR could be a predictor of tumor aggressiveness for ccRCC patients (Rausch et al. 2019).

p-mTOR, but not mTOR, was a poor prognostic factor of CSS in ccRCC patients. During 4

195

196

197

198

199

200

201

202

203

204

205

206

207

years follow-up, Kaplan-Meier survival curve displayed that CSS rate was higher in patients with low p-mTOR expression (score $<3$ ) than those with high expression $(\geq 3)(\log -$ rank=10.008, $P=0.002$ ), while CSS rate was not correlated with mTOR expression in 145 ccRCC patients (log-rank=1.373, $P=0.241$, Fig. 3 ). Then we further investigated the role of mTOR (including p-mTOR) on tumor prognosis using Cox regression analysis. Univariate survival analysis demonstrated that high p-mTOR expression was associated with a shorter CSS in patients with ccRCC [hazard ratio (HR) 2.933, 95\% confidence interval $(\mathrm{CI})=1.891-4.549$, $P<0.001$, Table 2]. In the meantime, it manifested that advanced stage (HR 4.869, 95\% CI $=$ 3.285-7.217, $P<0.001)$, high grade $(\mathrm{HR} 2.152,95 \% \mathrm{CI}=1.573-2.943, P<0.001)$, large tumor $(\mathrm{HR} 2.414,95 \% \mathrm{CI}=1.492-3.906, P<0.001)$, metastasis $(\mathrm{HR} 9.060, \mathrm{CI}=5.179-15.849, P<$ 0.001) were all correlated with poor prognosis for CSS. As for mTOR, its expression was not associated with CSS $(P=0.547)$. And CSS was not associated with patients' sex or age ( $P=$ $0.245, P=0.055$, respectively). 
208 Further multivariate survival analysis displayed that tumor stage (HR 3.812, 95\% CI $=2.460$ 209 5.907, $P<0.001$ ), grade (HR 2.187, 95\% CI = 1.526-3.133, $P<0.001)$, metastasis (HR 2.894, $21095 \% \mathrm{CI}=1.585-5.282, P=0.001)$ and $\mathrm{p}-\mathrm{mTOR}(\mathrm{HR} 1.733,95 \% \mathrm{CI}=1.037-2.897, P=0.036)$, 211 were all recognized as independent predictors for CSS in patients with ccRCC (Table 2), while 212 tumor size, mTOR expression, patients' age, or sex were not identified as prognostic factors $(P=$ $2130.134, P=0.122, P=0.472, P=0.170$, respectively, Table 2).

214 TIMER and TISIDB analyses revealed the relationship between mTOR and immune

215 infiltrates in ccRCC. ccRCC is an immunotherapy-sensitive tumor, and mTOR regulates tumor 216 immunity (Posadas et al. 2017; Saxton \& Sabatini 2017). On the base of the expression and 217 prognosis of mTOR in ccRCC, we further evaluated the correlation between mTOR and immune 218 features, such as immune cells and immunomodulators, in ccRCC using TIMER and TISIDB 219 databases. TIMER analysis showed that mTOR expression was positively correlated with 220 infiltrating levels of all the six TIIC subsets, i.e., macrophage (rho=0.419, $P<0.001$ ), neutrophil 221 (rho=0.432, $P<0.001$ ), dendritic cell (rho=0.368, $P<0.001)$, B cell (rho=0.214, $P<0.001), \mathrm{CD} 8+$ 222 T cell $(\mathrm{rho}=0.221, P<0.001)$, and $\mathrm{CD} 4+\mathrm{T}$ cell $(\mathrm{rho}=0.470, P<0.001)$ among the $533 \mathrm{ccRCC}$ 223 cases (Fig. 4). At the same time, TISIDB analysis demonstrated that mTOR expression was 224 significantly correlated to the abundance of numerous TILs in 534 ccRCC cases (Fig. 5). 225 Specifically, mTOR expression was positively related with the abundance of natural killer cell 226 (NK, rho $=0.258, P<0.001)$, regulatory $\mathrm{T}$ cell (Treg, $\mathrm{rho}=0.249, P<0.001)$, and was negatively 227 correlated with the abundance of CD56 bright natural killer cell (CD56bright, rho=-0.309, 228 $P<0.001)$ and CD56 dim natural killer cell (CD56dim, rho=-0.299, $P<0.001)$. Then we evaluated 229 the association between mTOR expression and the abundance of 24 immunoinhibitors across 230 human cancers, which was illustrated in Fig. 6. Particularly, the immunoinhibitors displaying the 231 greatest positively correlations included CD274 (PD-L1, rho=0.364, $P<0.001$ ), transforming 232 growth factor- $\beta$ receptor type I (TGFBR1, rho=0.290, $P<0.001$ ), colony stimulating factor-1 233 receptor $(\mathrm{CSF} 1 \mathrm{R}, \mathrm{rho}=0.254, P<0.001)$, while PDCD1 (PD-1) was not significantly associated 234 with mTOR in $\operatorname{ccRCC}$ ( $\mathrm{rho}=-0.064, P=0.137)$. HE staining demonstrated that the immune cells 
235 were sporadically scattered in the cancerous tissues, especially in the tissues with higher p236 mTOR expression (Supplementary Figure 1), which confirmed the rich immune infiltrates in the 237 ccRCC tissues (Diaz-Montero et al. 2020). The above results implied that mTOR could be 238 involved in regulating the immune infiltrates in ccRCC patients, which was consistent with 239 previous reports (Diaz-Montero et al. 2020; Zhang et al. 2019).

\section{Discussion}

$241 \mathrm{PI} 3 \mathrm{~K} / \mathrm{AKT} / \mathrm{mTOR}$ pathway plays a pivotal role in cancer pathogenesis and progression, and 242 mTOR is frequently activated in various malignancies, such as breast, prostate, lung, liver, and 243 kidney cancers (Posadas et al. 2017). Increasing literature has elaborated mTOR activation in 244 RCC, while its clinical value during RCC pathogenesis has not been fully elucidated yet (Cancer 245 Genome Atlas Research 2013; Chen et al. 2016; Darwish et al. 2013; Han et al. 2017; Zhang et 246 al. 2017). Our previous study revealed that expression of mTOR pathway members might play 247 distinguished roles in different stages or grades of RCC, implying the complexity of mTOR 248 signaling pathway in the development of RCC (Fan et al. 2020). Thus, it is necessary to present a 249 better looking of the relationship between mTOR signaling pathway and RCC. In the current 250 study, we compared the expression level of mTOR and p-mTOR between ccRCC and adjacent 251 kidney tissues, evaluated its prognostic significance using TMA, then revealed the tumor252 immune interaction of mTOR in ccRCC using online databases (TIMER and TISIDB) and HE 253 staining. Our study validated mTOR activation in ccRCC, i.e., the ratio of p-mTOR to mTOR 254 was increased in ccRCC $(P<0.05)$, which was also observed in previous report (Kruck et al. 255 2010). Subsequently IHC analysis demonstrated that p-mTOR expression was positively 256 correlated with tumor size, pathological stage, and metastasis status ( $P=0.001, P<0.001$, $257 P=0.003$, respectively), and reversely correlated with CSS $(\mathrm{P}<0.001)$, which implied elevated $\mathrm{p}-$ 258 mTOR was significantly correlated with the aggressiveness of ccRCC (Rausch et al. 2019). Then 259 survival analysis elucidated that p-mTOR was identified as an independent predictor of poor 260 CSS in 145 patients with ccRCC $(\mathrm{P}<0.05)$. Further TIMER and TISIDB databases analysis revealed mTOR could regulate numerous immune cells (including TIICs and TILs) and 
262 immunoinhibitors in $>500 \mathrm{ccRCC}$ patients $(P<0.001$, respectively), and HE staining revealed the 263 close relationship between p-mTOR expression and the infiltration of immune cells. The above 264 results also provided rationale for mTOR-targeted therapy of RCC, especially metastatic RCC. 265 To our knowledge, this is the first report that evaluates the prognostic role and interaction to 266 immune infiltration of mTOR in ccRCC patients simultaneously, which revealed p-mTOR could 267 be a prognostic factor and mTOR regulate multiple immune features in ccRCC patients.

268 Extensive literature reported mTOR was implicated in the initiation and progression of RCC 269 (Darwish et al. 2013; Kruck et al. 2010; Liontos et al. 2017; Rausch et al. 2019).

270 Kruck compared the expression profiles of $m$ TOR and p-mTOR (S2448) in ccRCC and adjacent 271 kidney tissues $(n=10)$, which found the ratio of $\mathrm{p}$-mTOR to mTOR was increased in 272 ccRCC (Kruck et al. 2010), which was also observed in the present study. A subsequent TMA 273 analysis revealed that elevated p-mTOR, instead of mTOR, was associated with tumor 274 aggressiveness and impaired OS in 342 primary and 90 metastatic ccRCC patients, and univariate 275 survival analysis displayed that elevated p-mTOR was a predictive marker of poor OS (Rausch et al. 276 2019), which validated our present study. Furthermore, Liontos reported that combination of 277 increased p-mTOR and low vascular endothelial growth factor (VEGF) expression was 278 negatively associated with OS in 79 patients with $\mathrm{mRCC}$ who were refractory to first-line 279 sunitinib treatment (Liontos et al. 2017). Darwish evaluated the prognostic significance of multiple 280 biomarkers in mTOR pathway components, including p-mTOR in 419 patients with ccRCC, and found a 281 negative correlation between the marker score of mTOR pathway components and the recurrence-free 282 survival (RFS) and CSS in nonmetastatic ccRCC patients (Darwish et al. 2013). Notably, p-mTOR, but 283 not mTOR, was also associated with aggressive features and poor prognosis in multiple cancers, such as 284 lung, live and esophagus (Hirashima et al. 2010; Lu et al. 2020; Su et al. 2016). These literatures revealed 285 the negative role of p-mTOR in the patient's survival. Through large-scale proteogenomic analysis, 286 several researches demonstrated mTOR alteration or activation in RCC, but most of them were 287 not involved in its prognosis (Cancer Genome Atlas Research 2013; Chen et al. 2016; Fan et al. 288 2020; Han et al. 2017; Zhang et al. 2017). Using integrative proteogenomic analysis, 
289 Zhang found that $\mathrm{p}-\mathrm{mTOR}$ expression level was significantly correlated with improved outcomes 290 in 32 major types of cancer including RCC, which was contradictory to the experimental results 291 (Zhang et al. 2017). Similarly, we reported the prognosis of PI3K/AKT/mTOR pathway 292 members in ccRCC using public databases analysis, which demonstrated that mTOR mRNA 293 were positively correlated with OS of over 500 ccRCC patients, and p-mTOR (S2448) was also 294 positively correlated with OS in patients with ccRCC $(n=445)$ (Fan et al. 2020). But no 295 experimental evidence has validated the positive prognosis of mTOR in ccRCC patients yet. 296 Collectively, the prognostic role of mTOR in RCC remains controversial. The prognostic 297 inconsistency of mTOR in RCC might be due to RCC specimens (remarkable heterogeneity, 298 varied sample size and divergence of clinicopathological backgrounds), mutation and 299 amplification of PI3K/AKT/mTOR pathway members, or overexpression of the components of 300 mTORC1 and mTORC2 (Fan et al. 2020; Hua et al. 2019).

301 Human immune system could recruit and activate immune guardian-T cells to identify and 302 eliminate malignant cells through antigen-antibody response and cell-mediated immunotoxicity. 303 To this end, tumor immune microenvironment plays a determinative role in tumor survival and 304 progression through mediating the anti-tumor immune response (Posadas et al. 2017; Saxton \& 305 Sabatini 2017). Comprehensive investigation of tumor and immune infiltrates would assist to 306 elucidate cancer pathogenesis and develop novel immunotherapy strategies (Ru et al. 2019). 307 ccRCC is recognized as one of the more responsive tumors to immunotherapy, and mTOR has 308 been reported to be participate in tumor immunity (Posadas et al. 2017; Saxton \& Sabatini 2017). 309 Recent studies reported that mTOR could regulate tumor immunity through modulating the interactions 310 between the stroma and the tumor, thus possibly promote carcinogenesis (Guri et al. 2018; Irelli et al. 311 2019). mTOR not only regulated the innate and adaptive immune response through modulating the 312 effector response of innate immune cells such as macrophage, DCs, neutrophils, and NKs, but also played 313 a prerequisite role in the development of adaptive immune cells, such as CD4+ T, CD8+ T, Tregs and B 314 cells (Nazari et al. 2021). The underlying mechanism behind the association of mTOR expression and 315 immune cells has not been clearly illustrated. Briefly, mTOR could regulate the expression of 
316 cytokines/chemokines, including interleukin-10 (IL-10) and transforming growth factor- $\beta$ (TGF- $\beta$ ), 317 and/or membrane receptors, such as cytotoxic T-Lymphocyte protein 4 (CTLA-4), PD-L1 and PD-1, to

318 modulate tumor immune cells (Irelli et al. 2019). In addition, Donnelly demonstrated that mTOR 319 activity was prerequisite for the production of interferon- $\gamma$ (IFN- $\gamma)$, a key NK cell effector molecule, and 320 IFN- $\gamma$ subsequently activated NK cell to regulate immune responses (Donnelly et al. 2014). Moreover, 321 Sordi reported inhibition of mTORC1 by rapamycin could increase the migration of DCs to lymph nodes 322 in vivo through upregulating CC-chemokine receptor 7 (CCR7) expression (Sordi et al. 2006). Immune 323 checkpoint inhibitor represented by PD-1 and PD-L1 monoclonal antibodies has been

324 325 recommended for mRCC patients. PD-1 (also called PDCD1, CD279) is expressed on immune cells, especially tumor specific T cells, while PD-1 ligand PD-L1 (also called B7-H1, CD274) is expressed on tumor cells as an "adaptive immune mechanism" to escape anti-tumor response (Han et al. 2020). In the current study, we investigated the relationship between mTOR and immune infiltration in ccRCC. Our data demonstrated mTOR was not only significantly associated with infiltrating levels of numerous immune cell subtypes such as NK and Treg cells, but also positively correlated with various immunoinhibitors such as CD274 (PD-L1) in ccRCC (Saxton \& Sabatini 2017). What's more, we also detected rich immune infiltration in ccRCC tissues using HE staining (Diaz-Montero et al. 2020), especially in the tissues with higher mTOR expression, which demonstrated that mTOR expression was closely associated with intratumoral immune infiltration. These results indicated the underlying mechanism of mTOR in ccRCC development might be involved in tumor immunity, such as immune cells and immunomodulator PD-L1.

In addition to intratumoral immune cells, recent studies began to uncovered the role of inflammatoryrelated parameters in peripheral blood, such as neutrophil and lymphocyte, during RCC development. There were increasing evidences that inflammatory-related parameters were of prognostic role in $\mathrm{mRCC}$ patients. Neutrophilia and thrombocytosis are markers of systemic inflammation, while lymphopenia is related to dysfunctions of the immune system. Kucharz evaluated inflammatory parameters, such as neutrophil, lymphocyte and platelet in $131 \mathrm{mRCC}$ patients treated with Sunitinib, and found that a

Peer] reviewing PDF | (2021:04:60110:1:1:NEW 17 Jun 2021) 
343 neutrophil-to-lymphocyte ratio (NLR) lower than 1.64 predicted better outcomes (PFS, OS) (Kucharz et

344 al. 2019). Similarly, Tjokrowidjaja reported enhanced NLR was associated with shorter survival adjusting

345 for Memorial Sloan Kettering Cancer Center (MSKCC) variables in $1102 \mathrm{mRCC}$ patients (Tjokrowidjaja

346 et al. 2020). To the best of our knowledge, there has been no report about the relationship between mTOR

347 and blood inflammatory cells in RCC yet, and further studies are needed to clarify this issue.

348 There are some limitations of this study. The first one is the limited sample size for prognostic

349 analysis, and more specimens or even multiple-center clinical studies should be needed for

350 further prospective validation. The second limitation is that only primary ccRCC tissues were

351 enrolled in the present study, and metastatic lesions, such as adrenal gland, lymph node and lung,

352 of mRCC patients, are not included. The prognostic significance and tumor-immune interaction

353 between primary and metastatic lesions of the same patients should be analyzed. Finally, it

354 should be marked that the expression patterns of adjacent renal tissues from RCC patients might

355 differ from benign renal tissue from healthy patients.

356

357 Conclusions

358 In summary, the present study detected the expression of mTOR and p-mTOR in ccRCC patients

359 using WB analysis, investigated their prognosis using IHC assay, and explored the interactions

360 between mTOR and immune infiltrates using TIMER and TISIDB databases, which aimed to

361 elucidate the clinical significance of mTOR in ccRCC. We found the ratio of p-mTOR to mTOR

362 was higher in ccRCC than adjacent tissues, p-mTOR was identified as a poor prognostic factor of

363 CSS, and mTOR was associated with numerous immune features in ccRCC patients, which

364 validated mTOR could be implicated in the initiation and progression of ccRCC.

365

366 Acknowledgements

367 Not applicable. 
369

370

371

372

373

374

375

376

377

378

379

380

381

382

383

384

385

386

387

388

389

390

391

392

393

394

395

396

397

398

399

400

401

402

403

404

405

406

407

408

\section{References}

Barata PC, and Rini BI. 2017. Treatment of renal cell carcinoma: Current status and future directions. $C A$ Cancer J Clin 67:507-524. 10.3322/caac.21411

Cancer Genome Atlas Research N. 2013. Comprehensive molecular characterization of clear cell renal cell carcinoma. Nature 499:43-49. 10.1038/nature12222

Chen F, Zhang Y, Senbabaoglu Y, Ciriello G, Yang L, Reznik E, Shuch B, Micevic G, De Velasco G, Shinbrot E, Noble MS, Lu Y, Covington KR, Xi L, Drummond JA, Muzny D, Kang H, Lee J, Tamboli P, Reuter V, Shelley CS, Kaipparettu BA, Bottaro DP, Godwin AK, Gibbs RA, Getz G, Kucherlapati R, Park PJ, Sander C, Henske EP, Zhou JH, Kwiatkowski DJ, Ho TH, Choueiri TK, Hsieh JJ, Akbani R, Mills GB, Hakimi AA, Wheeler DA, and Creighton CJ. 2016. Multilevel Genomics-Based Taxonomy of Renal Cell Carcinoma. Cell Rep 14:2476-2489. 10.1016/j.celrep.2016.02.024

Darwish OM, Kapur P, Youssef RF, Bagrodia A, Belsante M, Alhalabi F, Sagalowsky AI, Lotan Y, and Margulis V. 2013. Cumulative number of altered biomarkers in mammalian target of rapamycin pathway is an independent predictor of outcome in patients with clear cell renal cell carcinoma. Urology 81:581-586. 10.1016/j.urology.2012.11.030

Diaz-Montero CM, Rini BI, and Finke JH. 2020. The immunology of renal cell carcinoma. Nat Rev Nephrol 16:721-735. 10.1038/s41581-020-0316-3

Donnelly RP, Loftus RM, Keating SE, Liou KT, Biron CA, Gardiner CM, and Finlay DK. 2014. mTORC1-dependent metabolic reprogramming is a prerequisite for NK cell effector function. $J$ Immunol 193:4477-4484. 10.4049/jimmunol.1401558

Fan D, Liu Q, Wu F, Liu N, Qu H, Yuan Y, Li Y, Gao H, Ge J, Xu Y, Wang H, Liu Q, and Zhao Z. 2020. Prognostic significance of PI3K/AKT/ mTOR signaling pathway members in clear cell renal cell carcinoma. PeerJ 8:e9261. 10.7717/peerj.9261

Guri Y, Nordmann TM, and Roszik J. 2018. mTOR at the Transmitting and Receiving Ends in Tumor Immunity. Front Immunol 9:578. 10.3389/fimmu.2018.00578

Han G, Zhao W, Song X, Kwok-Shing Ng P, Karam JA, Jonasch E, Mills GB, Zhao Z, Ding Z, and Jia P. 2017. Unique protein expression signatures of survival time in kidney renal clear cell carcinoma through a pan-cancer screening. BMC Genomics 18:678. 10.1186/s12864-017-4026-6

Han Y, Liu D, and Li L. 2020. PD-1/PD-L1 pathway: current researches in cancer. Am J Cancer Res 10:727-742.

Hirashima K, Baba Y, Watanabe M, Karashima R, Sato N, Imamura Y, Hiyoshi Y, Nagai Y, Hayashi N, Iyama K, and Baba H. 2010. Phosphorylated mTOR expression is associated with poor prognosis for patients with esophageal squamous cell carcinoma. Ann Surg Oncol 17:2486-2493. 10.1245/s10434-010-1040-1

Hua H, Kong Q, Zhang H, Wang J, Luo T, and Jiang Y. 2019. Targeting mTOR for cancer therapy. $J$ Hematol Oncol 12:71. 10.1186/s13045-019-0754-1

Irelli A, Sirufo MM, Scipioni T, De Pietro F, Pancotti A, Ginaldi L, and De Martinis M. 2019. mTOR Links Tumor Immunity and Bone Metabolism: What are the Clinical Implications? Int J Mol Sci 20. $10.3390 /$ ijms 20235841 
409 Kruck S, Bedke J, Hennenlotter J, Ohneseit PA, Kuehs U, Senger E, Sievert KD, and Stenzl A. 2010.

410 Activation of mTOR in renal cell carcinoma is due to increased phosphorylation rather than 411 protein overexpression. Oncol Rep 23:159-163.

412 Kucharz J, Dumnicka P, Giza A, Demkow U, Kusnierz-Cabala B, Demkow T, and Wiechno P. 2019.

413 Radiological Response and Neutrophil-to-Lymphocyte Ratio as Predictive Factors for

414 Progression-Free and Overall Survival in Metastatic Renal Cell Carcinoma Patients Treated with

$415 \quad$ Sunitinib. Adv Exp Med Biol 1153:31-45. 10.1007/5584_2019_352

416 Li J, Lu Y, Akbani R, Ju Z, Roebuck PL, Liu W, Yang JY, Broom BM, Verhaak RG, Kane DW,

417 Wakefield C, Weinstein JN, Mills GB, and Liang H. 2013. TCPA: a resource for cancer

418 functional proteomics data. Nat Methods 10:1046-1047. 10.1038/nmeth.2650

419 Li T, Fan J, Wang B, Traugh N, Chen Q, Liu JS, Li B, and Liu XS. 2017. TIMER: A Web Server for

420 Comprehensive Analysis of Tumor-Infiltrating Immune Cells. Cancer Res 77:e108-e110.

$421 \quad 10.1158 / 0008-5472 . C A N-17-0307$

422 Liontos M, Trigka EA, Korkolopoulou P, Tzannis K, Lainakis G, Koutsoukos K, Kostouros E, Lykka M, Papandreou CN, Karavasilis V, Christodoulou C, Papatsoris A, Skolarikos A, Varkarakis I, Adamakis I, Alamanis C, Stravodimos K, Mitropoulos D, Deliveliotis C, Constantinidis CA, Saetta A, Patsouris E, Dimopoulos M, and Bamias A. 2017. Expression and prognostic significance of VEGF and mTOR pathway proteins in metastatic renal cell carcinoma patients: a prognostic immunohistochemical profile for kidney cancer patients. World J Urol 35:411-419. 10.1007/s00345-016-1890-7

Lu J, Zang H, Zheng H, Zhan Y, Yang Y, Zhang Y, Liu S, Feng J, Wen Q, Long M, and Fan S. 2020. Overexpression of p-Akt, p-mTOR and p-eIF4E proteins associates with metastasis and unfavorable prognosis in non-small cell lung cancer. PLoS One 15:e0227768. 10.1371/journal.pone.0227768

Makhov P, Joshi S, Ghatalia P, Kutikov A, Uzzo RG, and Kolenko VM. 2018. Resistance to Systemic Therapies in Clear Cell Renal Cell Carcinoma: Mechanisms and Management Strategies. Mol Cancer Ther 17:1355-1364. 10.1158/1535-7163.MCT-17-1299

Nazari N, Jafari F, Ghalamfarsa G, Hadinia A, Atapour A, Ahmadi M, Dolati S, and Rostamzadeh D. 2021. The emerging role of miRNA in regulating the mTOR signaling pathway in immune and inflammatory responses. Immunol Cell Biol. 10.1111/imcb.12477

Posadas EM, Limvorasak S, and Figlin RA. 2017. Targeted therapies for renal cell carcinoma. Nat Rev Nephrol 13:496-511. 10.1038/nrneph.2017.82

Rausch S, Schollenberger D, Hennenlotter J, Stuhler V, Kruck S, Stenzl A, and Bedke J. 2019. mTOR and mTOR phosphorylation status in primary and metastatic renal cell carcinoma tissue: differential expression and clinical relevance. J Cancer Res Clin Oncol 145:153-163. 10.1007/s00432-018-2775-5

Rini BI, Campbell SC, and Escudier B. 2009. Renal cell carcinoma. Lancet 373:1119-1132. 10.1016/S0140-6736(09)60229-4

Ru B, Wong CN, Tong Y, Zhong JY, Zhong SSW, Wu WC, Chu KC, Wong CY, Lau CY, Chen I, Chan NW, and Zhang J. 2019. TISIDB: an integrated repository portal for tumor-immune system interactions. Bioinformatics 35:4200-4202. 10.1093/bioinformatics/btz210 
450

451

452

453

454

455

456

457

458

459

460

461

462

463

464

465

466

467

468

469

470

471

472

473

474

475

476

477

478

479

480

481

482

483

484

485

Saxton RA, and Sabatini DM. 2017. mTOR Signaling in Growth, Metabolism, and Disease. Cell 169:361-371. 10.1016/j.cell.2017.03.035

Siegel RL, Miller KD, Fuchs HE, and Jemal A. 2021. Cancer Statistics, 2021. CA Cancer J Clin 71:7-33. 10.3322/caac. 21654

Sordi V, Bianchi G, Buracchi C, Mercalli A, Marchesi F, D'Amico G, Yang CH, Luini W, Vecchi A, Mantovani A, Allavena P, and Piemonti L. 2006. Differential effects of immunosuppressive drugs on chemokine receptor CCR7 in human monocyte-derived dendritic cells: selective upregulation by rapamycin. Transplantation 82:826-834. 10.1097/01.tp.0000235433.03554.4f

Su R, Nan H, Guo H, Ruan Z, Jiang L, Song Y, and Nan K. 2016. Associations of components of PTEN/AKT/mTOR pathway with cancer stem cell markers and prognostic value of these biomarkers in hepatocellular carcinoma. Hepatol Res 46:1380-1391. 10.1111/hepr.12687

Sung H, Ferlay J, Siegel RL, Laversanne M, Soerjomataram I, Jemal A, and Bray F. 2021. Global cancer statistics 2020: GLOBOCAN estimates of incidence and mortality worldwide for 36 cancers in 185 countries. CA Cancer J Clin. 10.3322/caac. 21660

Tjokrowidjaja A, Goldstein D, Hudson HM, Lord SJ, Gebski V, Clarke S, de Souza P, Motzer RJ, and Lee CK. 2020. The impact of neutrophil-lymphocyte ratio on risk reclassification of patients with advanced renal cell cancer to guide risk-directed therapy. Acta Oncol 59:20-27. 10.1080/0284186X.2019.1656342

Yuan Y, Yang X, Li Y, Liu Q, Wu F, Qu H, Gao H, Ge J, Xu Y, Wang H, Wang Y, and Zhao Z. 2020. Expression and prognostic significance of fatty acid synthase in clear cell renal cell carcinoma. Pathol Res Pract 216:153227. 10.1016/j.prp.2020.153227

Zhang C, Duan Y, Xia M, Dong Y, Chen Y, Zheng L, Chai S, Zhang Q, Wei Z, Liu N, Wang J, Sun C, Tang Z, Cheng X, Wu J, Wang G, Zheng F, Laurence A, Li B, and Yang XP. 2019. TFEB Mediates Immune Evasion and Resistance to mTOR Inhibition of Renal Cell Carcinoma via Induction of PD-L1. Clin Cancer Res 25:6827-6838. 10.1158/1078-0432.CCR-19-0733

Zhang Y, Kwok-Shing Ng P, Kucherlapati M, Chen F, Liu Y, Tsang YH, de Velasco G, Jeong KJ, Akbani R, Hadjipanayis A, Pantazi A, Bristow CA, Lee E, Mahadeshwar HS, Tang J, Zhang J, Yang L, Seth S, Lee S, Ren X, Song X, Sun H, Seidman J, Luquette LJ, Xi R, Chin L, Protopopov A, Westbrook TF, Shelley CS, Choueiri TK, Ittmann M, Van Waes C, Weinstein JN, Liang H, Henske EP, Godwin AK, Park PJ, Kucherlapati R, Scott KL, Mills GB, Kwiatkowski DJ, and Creighton CJ. 2017. A Pan-Cancer Proteogenomic Atlas of PI3K/AKT/mTOR Pathway Alterations. Cancer Cell 31:820-832 e823. 10.1016/j.ccell.2017.04.013

Zhao Z, Lu J, Han L, Wang X, Man Q, and Liu S. 2016. Prognostic significance of two lipid metabolism enzymes, HADHA and ACAT2, in clear cell renal cell carcinoma. Tumour Biol 37:8121-8130. $10.1007 / \mathrm{s} 13277-015-4720-4$

Peer) reviewing PDF | (2021:04:60110:1:1:NEW 17 Jun 2021) 
Figure 1

mTOR and p-mTOR expression between cCRCC and adjacent normal kidney tissues by WB analysis.

A. Relative expression levels of mTOR and p-mTOR in 3 pairs ccRCC (T) and their adjacent normal kidney (N) tissues. Left: WB bands; right: statistical bar chart. B. The ratio of p-mTOR to mTOR in ccRCC $(\mathrm{T})$ and normal kidney $(\mathrm{N})$ tissues. ${ }^{*} \mathrm{P}<0.05$.

A

T1 N1 T2 N2T3N3

mTOR

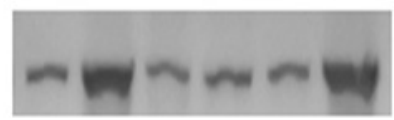

p-mTOR

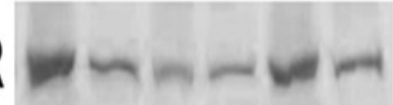

Actin
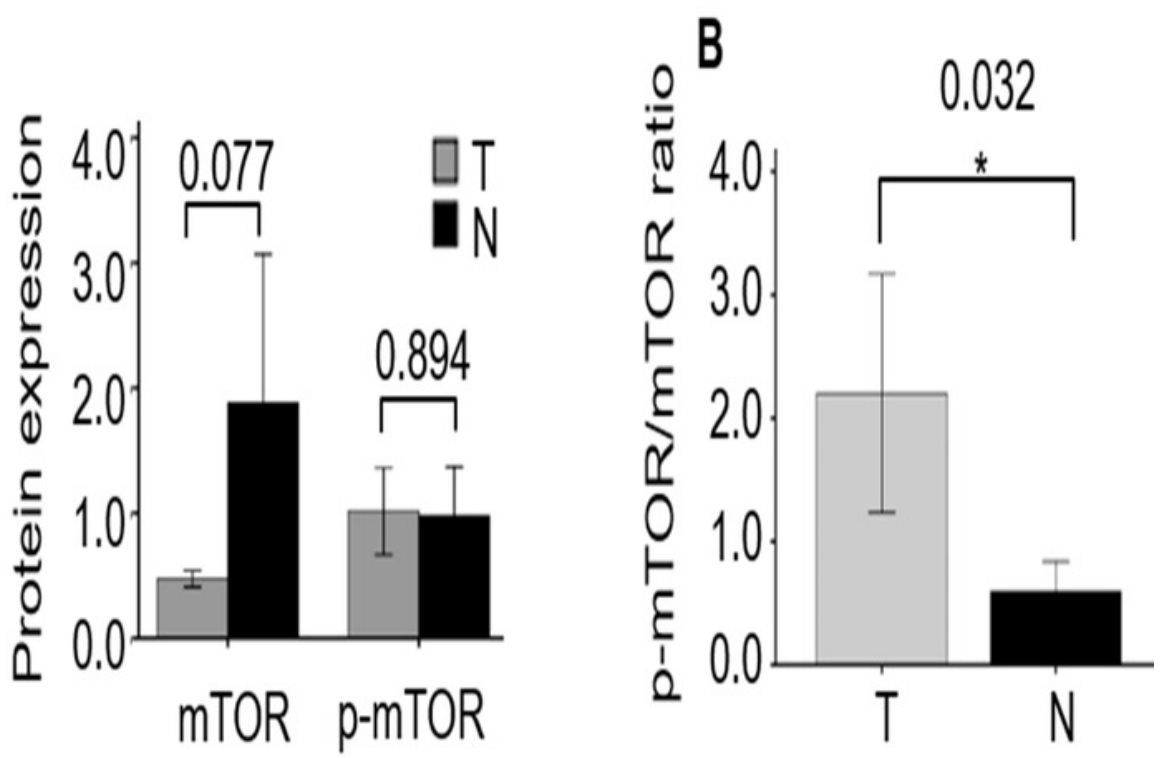


\section{Figure 2}

Representative immunostaining photomicrographs of mTOR and p-mTOR expression by IHC analysis.

Staining signals displayed cytoplasmic localization of mTOR in adjacent kidney (A, staining score 6) and ccRCC tissues (B: low expression, score 0, C: high expression, score 6), and pmTOR in adjacent kidney (D, score 4) and ccRCC tissues (E: low expression, score 3, F: high expression, score 7). The corresponding magnified areas in ccRCC and adjacent tissues also

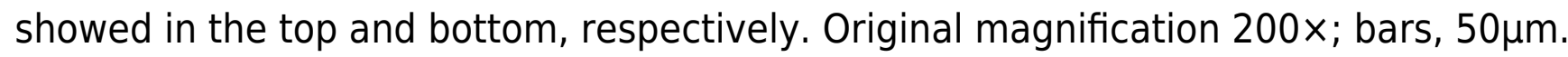


mTOR

\section{P-mTOR}

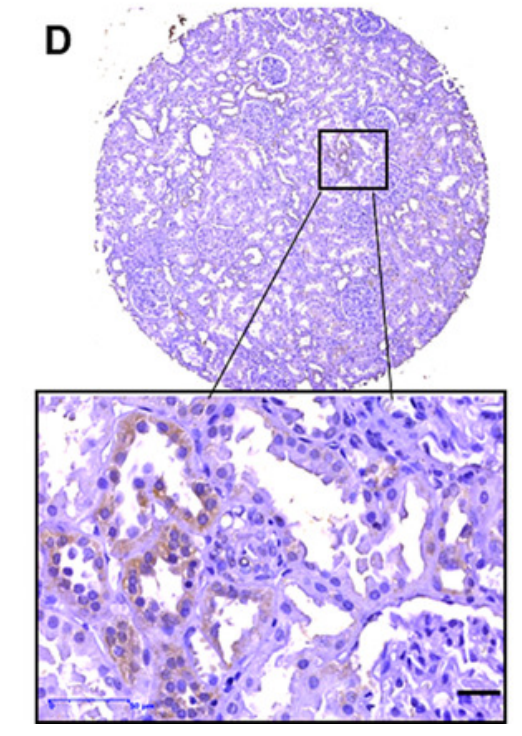

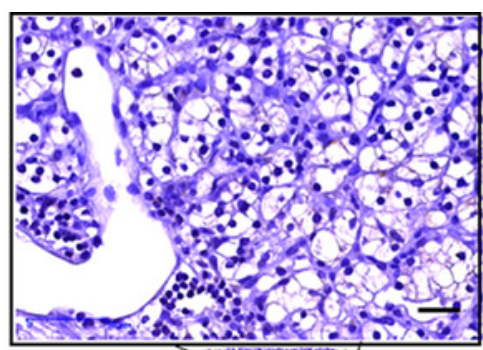

A
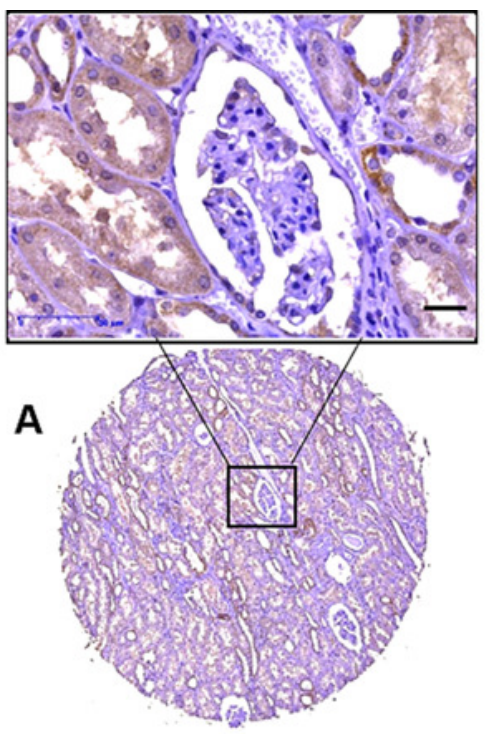

B

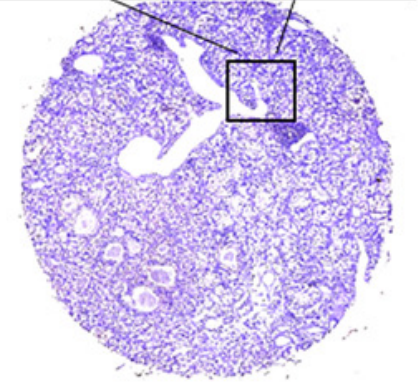

E
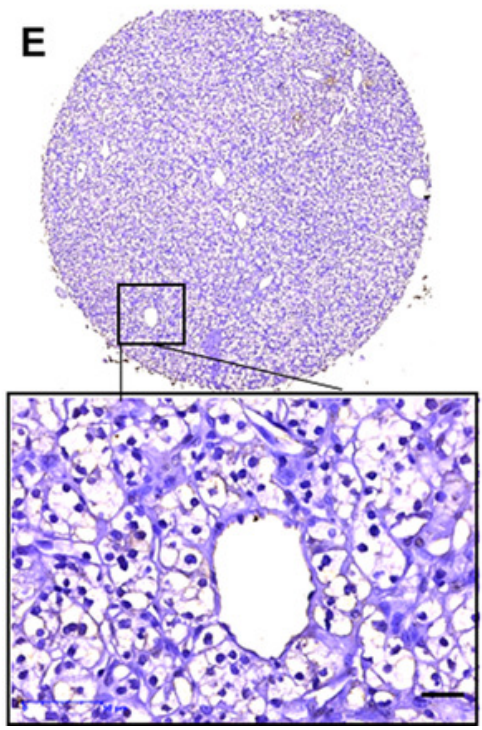
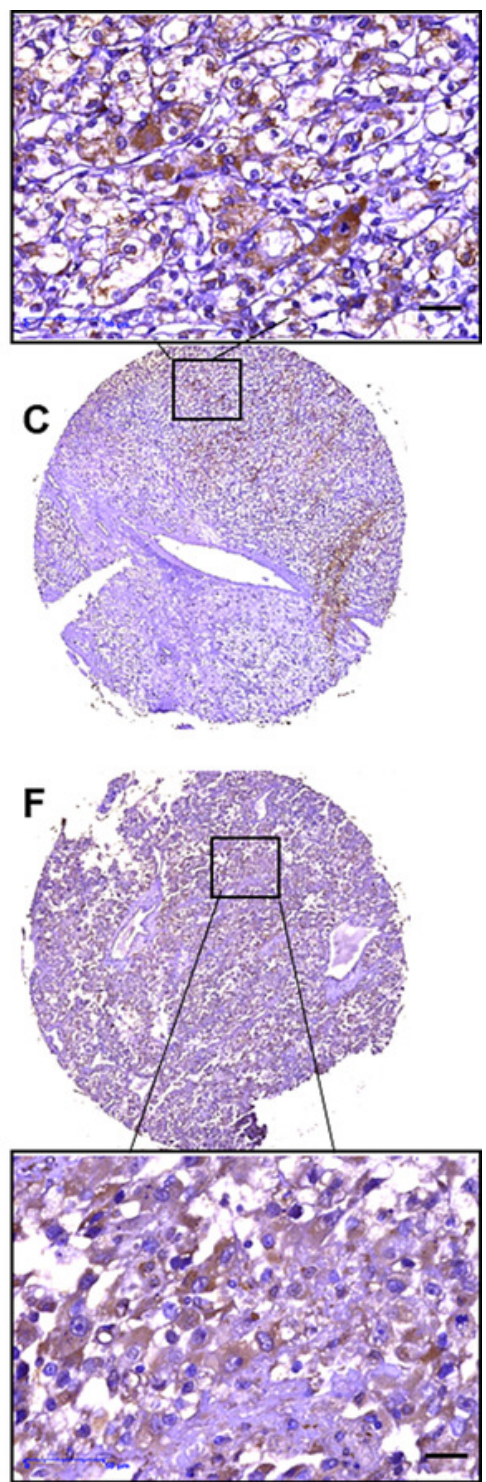
Figure 3

Kaplan-Meier survival curves demonstrated cancer-specific survival of 145 patients with ccRCC, according to mTOR (A) and p-mTOR (B) staining.

Blue and red curves represented high and low staining, respectively.
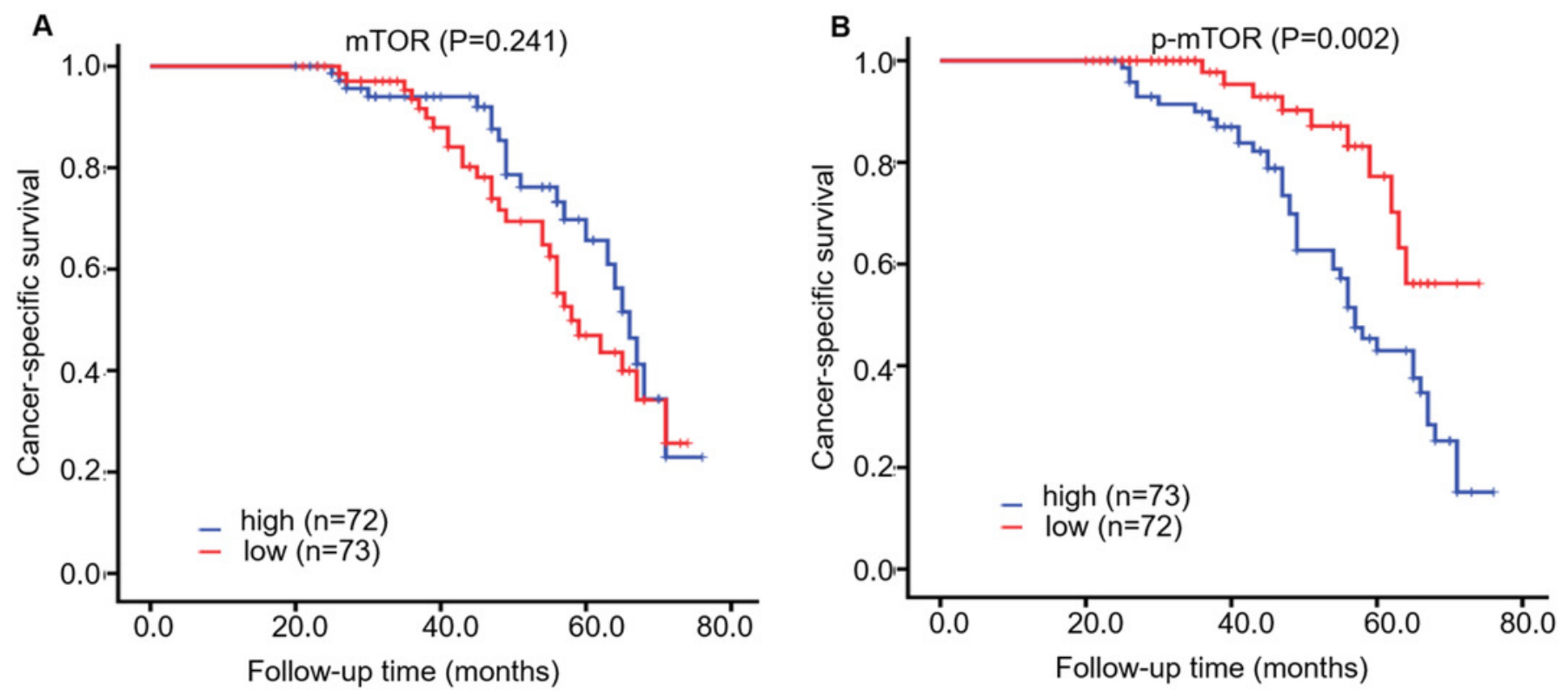
Figure 4

Correlation between mTOR expression and tumor-infiltrating immune cells (TIICs) in 533 cCRCC patients (TIMER).

The infiltration levels of the six TIIC subsets, i.e., macrophage (A), neutrophil (B), dendritic cell $(\mathbf{C})$, B cell $(\mathbf{D}), \mathrm{CD} 8+\mathrm{T}$ cell $(\mathbf{E})$, and CD4+ T cell (F). TPM: transcripts per million.
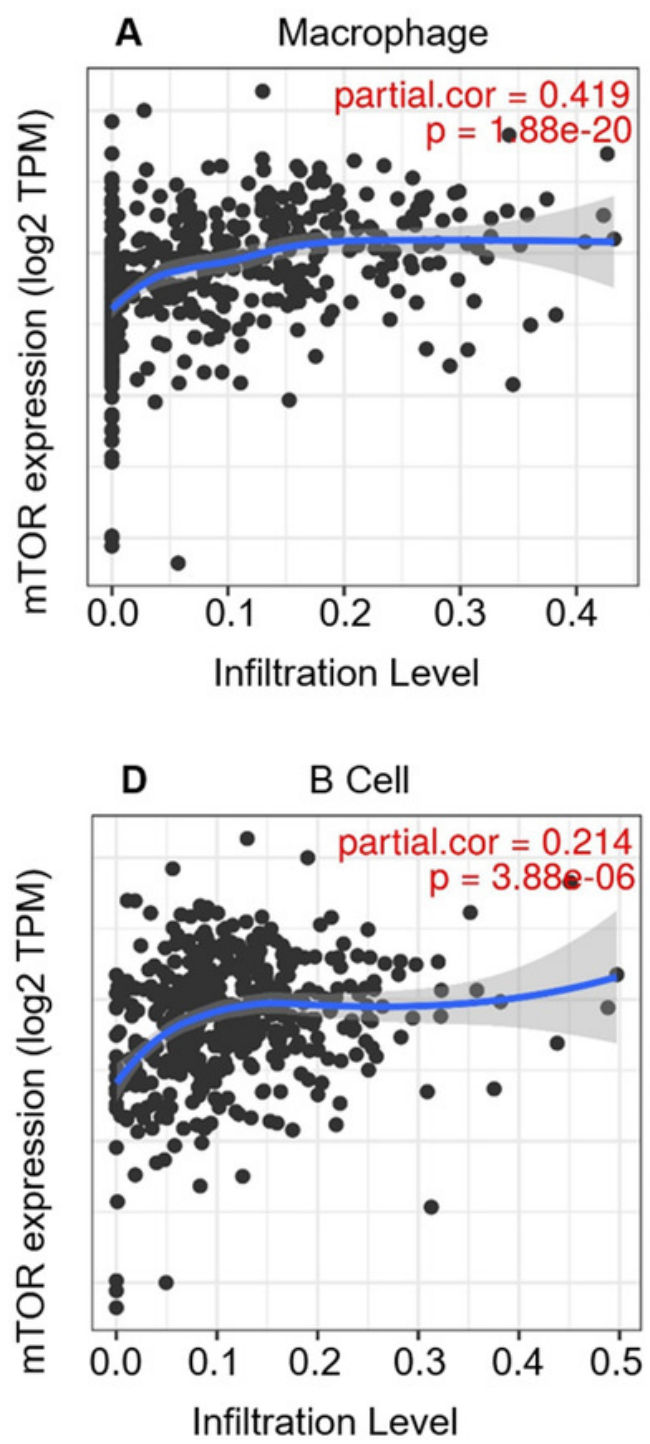
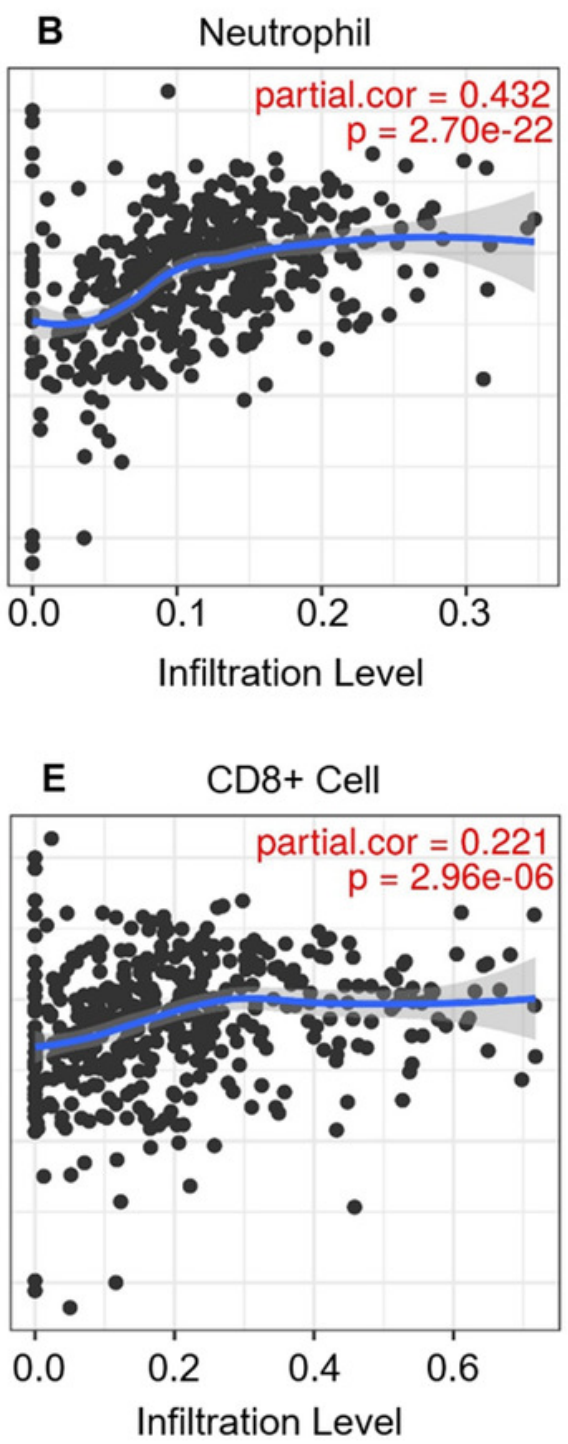

C Dendritic Cell
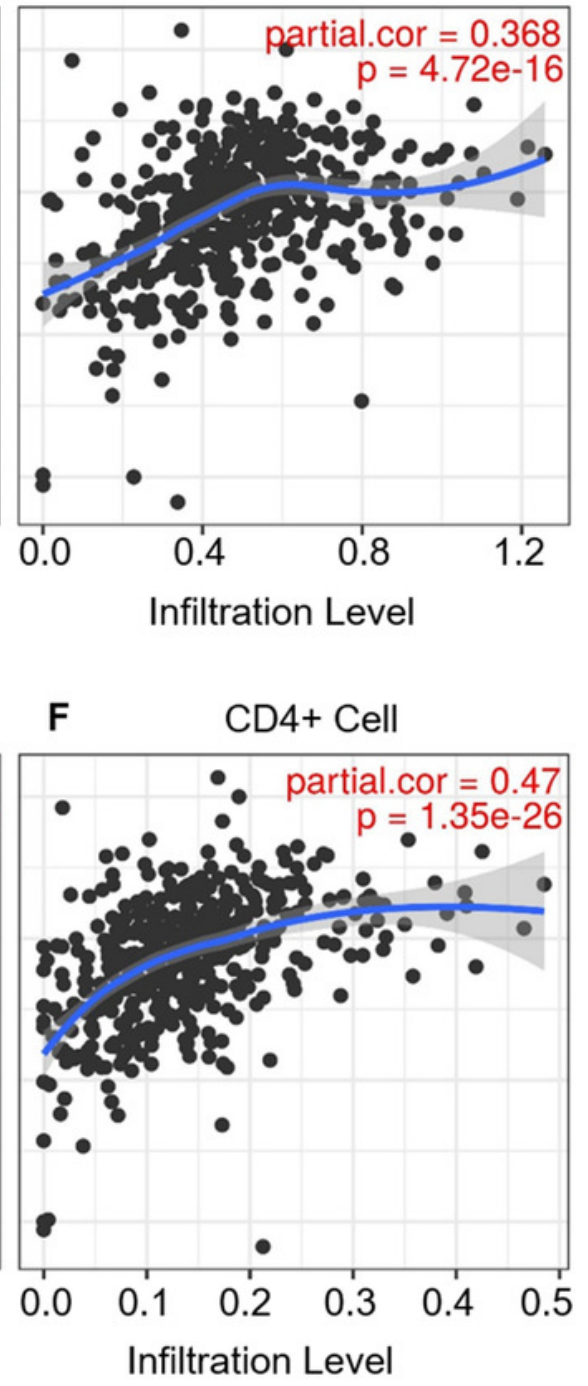


\section{Figure 5}

Correlation between mTOR expression and lymphocytes in 534 cCRCC patients (TISIDB).

A. The pan-cancer analysis of relationship between mTOR expression and abundance of the 28 tumor-infiltrating lymphocytes (TILS). The top 4 TILs either positive (B: NK cell, C: Treg cell) or negative (D: CD56bright cell; E: CD56dim cell) correlation with mTOR expression in cCRCC patients. ${ }^{*} P<0.05,{ }^{* *} P<0.01$.
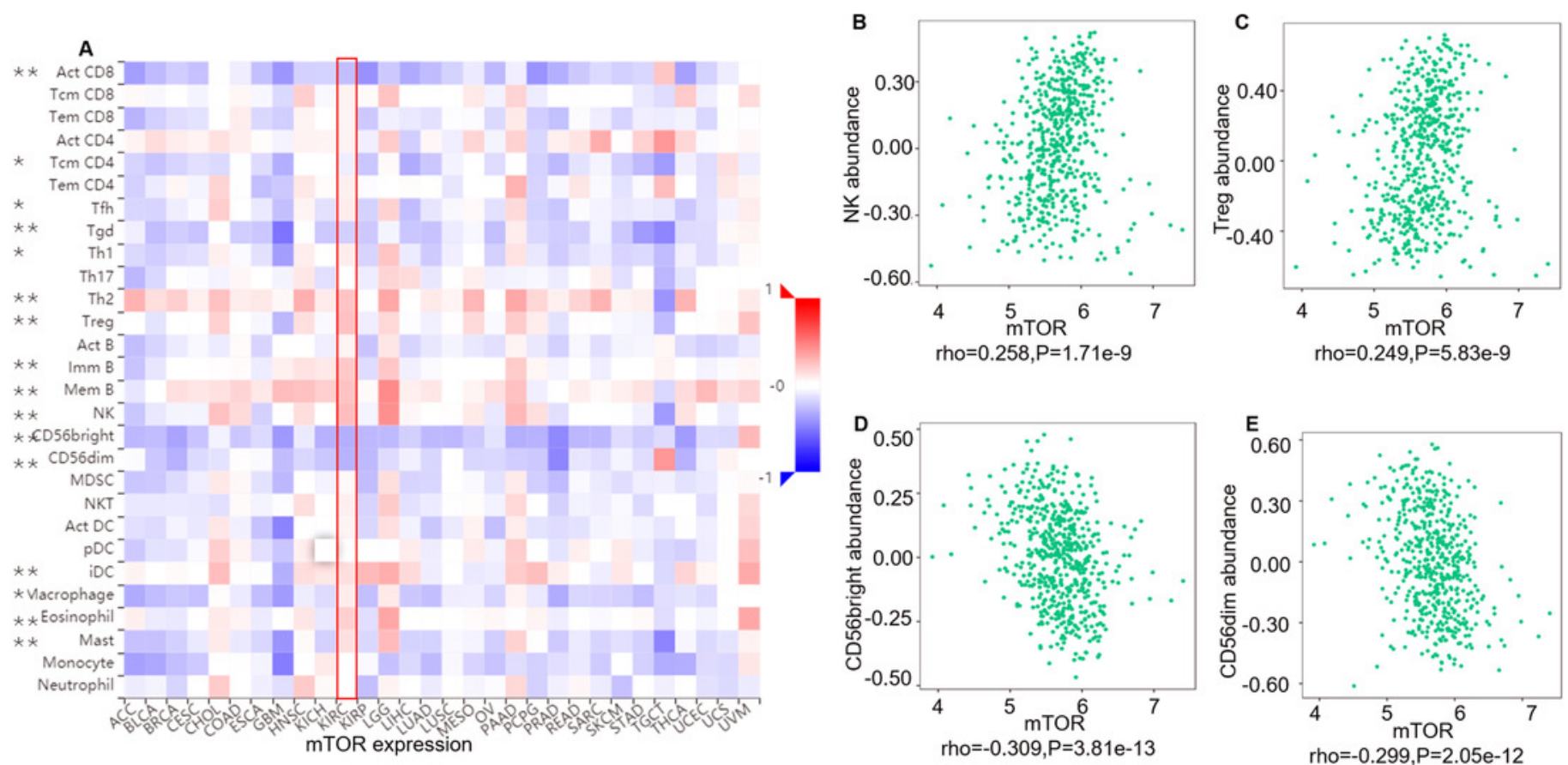
Figure 6

Correlation between mTOR expression and immunoinhibitors in $534 \mathrm{ccRCC}$ patients (TISIDB).

A. The pan-cancer analysis of relationship between mTOR expression and abundance of the 24 immunoinhibitors. The top 3 immunoinhibitors (B: CD274/PD-L1, C: TGFBR1, D: CSF1R) and PDCD1 (E, PD-1) correlation with mTOR expression in ccRCC patients. ${ }^{*} P<0.05,{ }^{* *} P<0.01$.
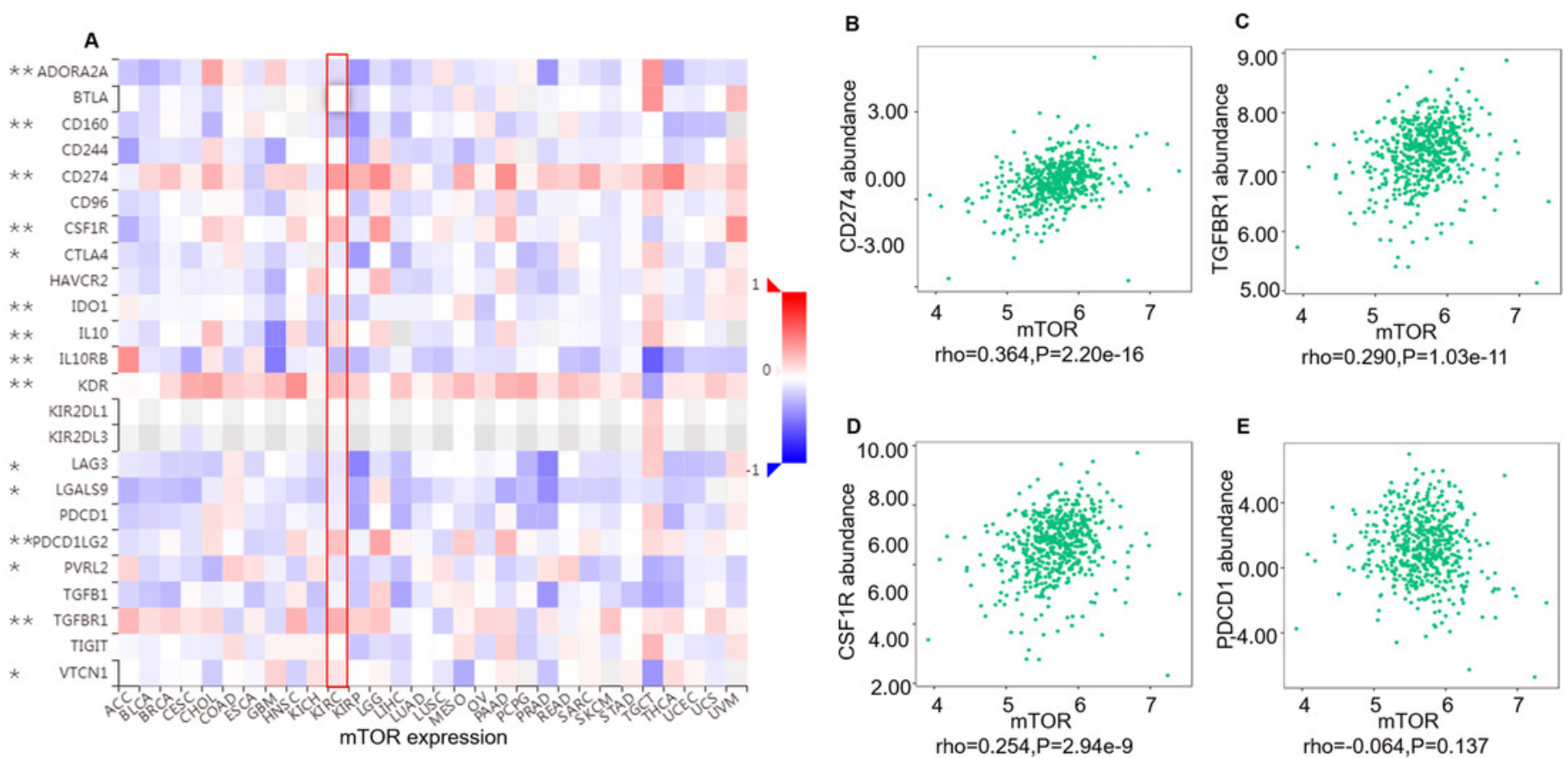


\section{Table 1 (on next page)}

Correlation between mTOR (including p-mTOR) expression and clinicopathological parameters of $\operatorname{ccRCC}(n=145)$ 
2 Table 1 Correlation between mTOR (including p-mTOR) expression and clinicopathological

3 parameters of $\operatorname{ccRCC}(\mathrm{n}=145)$

\begin{tabular}{|c|c|c|c|c|c|c|c|c|}
\hline \multirow{2}{*}{ Parameters } & \multicolumn{2}{|c|}{ p-mTOR staining } & \multirow[t]{2}{*}{$\chi^{2}$} & \multirow[t]{2}{*}{$P$-value } & \multicolumn{2}{|c|}{ mTOR staining } & \multirow[t]{2}{*}{$\chi^{2}$} & \multirow[t]{2}{*}{$P$-value } \\
\hline & Low, n (\%) & High, n (\%) & & & Low, n (\%) & High, n (\%) & & \\
\hline \multicolumn{9}{|l|}{ Sex } \\
\hline Male $(n=109)$ & $49(44.95)$ & $60(55.05)$ & & & $55(50.346)$ & $54(49.54)$ & & \\
\hline Female $(n=36)$ & 23(63.89) & $13(36.11)$ & 3.881 & 0.056 & $18(50.00)$ & $18(50.00)$ & 0.002 & 0.962 \\
\hline \multicolumn{9}{|l|}{ Age } \\
\hline$<60$ yrs $(\mathrm{n}=65)$ & $36(46.15)$ & $29(53.85)$ & & & $32(49.23)$ & $33(50.77)$ & & \\
\hline$\geq 60$ yrs $(n=80)$ & $36(45.00)$ & $44(55.00)$ & 1.547 & 0.244 & $41(51.25)$ & $39(48.75)$ & 0.058 & 0.868 \\
\hline \multicolumn{9}{|l|}{ ISUP grade } \\
\hline G $1(n=26)$ & $16(61.54)$ & $10(38.46)$ & & & $11(42.31)$ & $15(57.69)$ & & \\
\hline G $2(n=56)$ & $27(48.21)$ & $29(51.79)$ & & & $27(48.21)$ & $29(51.79)$ & & \\
\hline G 3-4 $(n=63)$ & $29(46.03)$ & $34(53.97)$ & 1.846 & 0.397 & $35(55.56)$ & $28(44.44)$ & 1.458 & 0.482 \\
\hline \multicolumn{9}{|l|}{ AJCC stage } \\
\hline T I $(n=42)$ & $33(78.57)$ & $9(21.43)$ & & & $22(52.38)$ & $20(47.62)$ & & \\
\hline T II (n=38) & $22(57.89)$ & $16(42.11)$ & & & $15(39.47)$ & $23(60.53)$ & & \\
\hline T III-IV (n=65) & $17(26.15)$ & $48(73.85)$ & 29.441 & $<0.001$ & $36(55.38)$ & $29(44.62)$ & 2.527 & 0.283 \\
\hline
\end{tabular}




\begin{tabular}{|c|c|c|c|c|c|c|c|c|}
\hline \multicolumn{9}{|l|}{ Tumor size } \\
\hline$<7.0 \mathrm{~cm}(\mathrm{n}=57)$ & $38(66.67)$ & $19(33.33)$ & & & $30(52.63)$ & $27(47.37)$ & & \\
\hline$\geq 7.0 \mathrm{~cm}(\mathrm{n}=88)$ & $34(38.64)$ & $54(61.36)$ & 10.872 & 0.001 & $43(48.86)$ & $45(51.14)$ & 0.196 & 0.735 \\
\hline \multicolumn{9}{|l|}{ Metastasis } \\
\hline Negative $(\mathrm{n}=121)$ & $67(55.37)$ & $54(44.63)$ & & & $60(49.59)$ & $61(50.41)$ & & \\
\hline Positive $(\mathrm{n}=24)$ & $5(20.83)$ & $19(79.17)$ & 9.557 & 0.003 & $13(54.17)$ & $11(45.83)$ & 0.168 & 0.824 \\
\hline \multicolumn{9}{|l|}{ Survival rate } \\
\hline Alive $(\mathrm{n}=51)$ & 41(80.39) & $10(19.61)$ & & & $22(43.14)$ & $29(56.86)$ & & \\
\hline Dead $(n=94)$ & $31(32.98)$ & $63(67.02)$ & 29.731 & $<0.001$ & $51(54.26)$ & $43(45.74)$ & 1.635 & 0.201 \\
\hline
\end{tabular}

4

5 Note: Statistical analyses were performed using Pearson chi-Square tests.

6 Abbreviation: ISUP: International Society of Urological Pathology, AJCC: American Joint Committee on Cancer. 


\section{Table 2 (on next page)}

Univariate and multivariate survival analysis of cancer-specific survival $(n=145)$ 
1 Table 2 Univariate and multivariate survival analysis of cancer-specific survival $(n=145)$

2

3

4

5

7

\begin{tabular}{|c|c|c|c|c|}
\hline Parameters & $\begin{array}{c}\text { Univariate }^{\mathrm{a}} \\
\mathrm{HR}(95 \% \mathrm{CI})^{\mathrm{b}} \\
\end{array}$ & $P$-value & $\begin{array}{c}\text { Multivariate }^{\mathrm{a}} \\
\text { HR }(95 \% \mathrm{CI})^{\mathrm{b}} \\
\end{array}$ & $P$-value \\
\hline Sex & $1.305(0.834-2.042)$ & 0.245 & $1.452(0.892-2.364)$ & 0.134 \\
\hline Age & $1.511(0.990-2.306)$ & 0.055 & $1.438(0.908-2.279)$ & 0.122 \\
\hline Grade $(\mathrm{G} 3-4)^{\mathrm{c}}$ & $2.152(1.573-2.943)$ & $<0.001$ & $2.187(1.526-3.133)$ & $<0.001$ \\
\hline Stage (TIII-IV) ${ }^{\mathrm{d}}$ & $4.869(3.285-7.217)$ & $<0.001$ & $3.812(2.460-5.907)$ & $<0.001$ \\
\hline Size $(\geq 7.0 \mathrm{~cm})$ & $2.414(1.492-3.906)$ & $<0.001$ & $1.216(0.713-2.073)$ & 0.472 \\
\hline Metastasis & $9.060(5.179-15.849)$ & $<0.001$ & $2.894(1.588-5.282)$ & 0.001 \\
\hline High p-mTOR & $2.933(1.891-4.549)$ & $<0.001$ & $1.733(1.037-2.897)$ & 0.036 \\
\hline High mTOR & $0.882(0.586-1.327)$ & 0.547 & $1.365(0.876-2.128)$ & 0.170 \\
\hline
\end{tabular}

proportional hazards regression model.

${ }^{\mathrm{b}}$ Abbreviation: HR: hazard ratio, CI: confidence interval.

${ }^{\mathrm{c}}$ For grade: 1, 2 vs 3-4.

${ }^{\mathrm{d}}$ For stage: I, II vs III-IV.

19 